\title{
Haptic Dimensions of Human-Robot Interaction
}

\author{
ALLISON M. OKAMURA, Department of Mechanical Engineering, Stanford University, USA
}

\author{
CCS Concepts: • Human-centered computing $\rightarrow$ Haptic devices; • Computer systems organization \\ $\rightarrow$ Robotics; • Hardware $\rightarrow$ Haptic devices; \\ Additional Key Words and Phrases: Wearable haptics
}

\section{ACM Reference format:}

Allison M. Okamura. 2018. Haptic Dimensions of Human-Robot Interaction. ACM Trans. Hum.-Robot Interact. 7, 1, Article 6 (May 2018), 3 pages.

https://doi.org/10.1145/3209768

Human manipulation, exploration, guidance, and interaction are all facilitated by haptics - the sense of touch. In the haptics research community, we strive to enable such activities in autonomous robots; there is a long history of research seeking to enable autonomous robots to sense, perceive, and reason using touch. Similarly, humans performing actions in virtual and augmented reality desire the enhanced realism provided by haptic feedback, especially when learning physical tasks that will later be performed in the real world. When humans control remote robots to do their bidding, haptics contributes to a sense of telepresence for the human operator and enables performance of physical tasks remote in distance or scale that are not yet feasible with autonomous robots. Compared to these traditional areas of haptics research, there is relatively little attention paid in the haptics research community to the kinds of scenarios most commonly associated with human-robot interaction: a human and robot, each a separate agent, seeking to collaborate with, learn from, or assist the other.

Many problems that feature this kind of haptic human-robot interaction remain unsolved because they are so multifaceted and interdisciplinary. Consider the classic problem of a human and robot working together to physically lift and move a piece of furniture. While this problem is considered solved in some respects, making this a reality that integrates the myriad aspects of human-robot interaction reveals how much is left to be done. The robot must be physically capable of performing the task, no small feat if the robot is also meant to be of a size practical for a domestic environment, and it must be materially safe for working in close proximity with humans. The human and robot must each be able to develop high-level plans based on uncertain information, communicate intent, and decide at a low level when and how to act. Communication and interaction require choosing the appropriate modality for accuracy and efficiency. While visual and verbal/auditory communication are commonly used by both humans and robots, the physical component of a collaborative furniture-moving task means that haptic interaction is also inherently present. As social roboticists are well aware, robot behavior and interaction strategies greatly effect usability and acceptance; this is just as true for haptics as it is for vision and audition.

Authors' address: A. M. Okamura, Department of Mechanical Engineering, Stanford University, Stanford, CA, 94305, USA. Permission to make digital or hard copies of part or all of this work for personal or classroom use is granted without fee provided that copies are not made or distributed for profit or commercial advantage and that copies bear this notice and the full citation on the first page. Copyrights for third-party components of this work must be honored. For all other uses, contact the Owner/Author.

2018 Copyright is held by the owner/author(s).

2573-9522/2018/05-ART6

https://doi.org/10.1145/3209768 


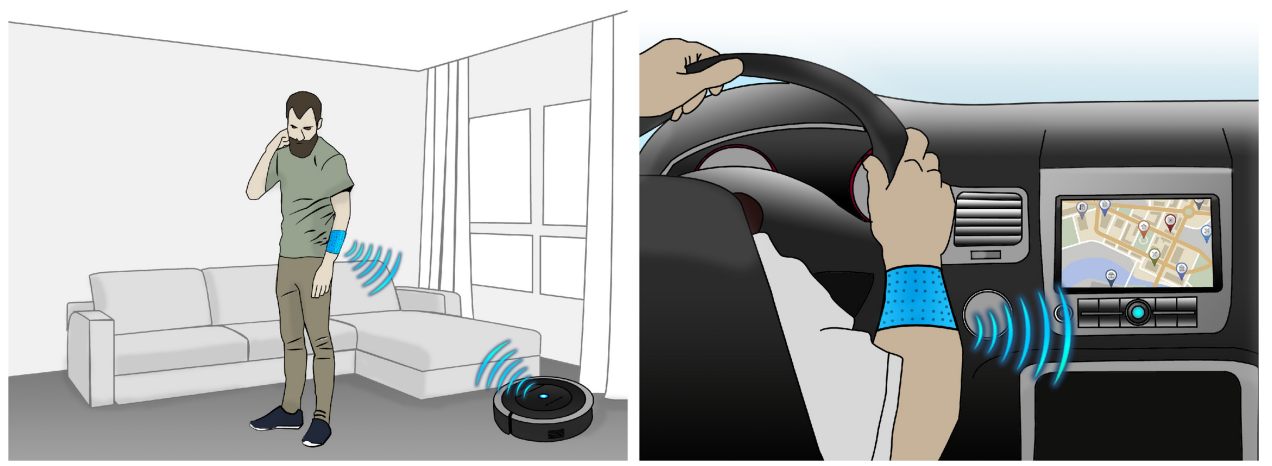

Fig. 1. A human wears a tactile display on the arm that conveys and receives information from an autonomous robot, which could range from a mobile robot in a domestic environment to an autonomous car handing over control. Wearable tactile displays can cleave the overloaded role of haptics that occurs when direct physical contact between the human and robot results in haptics being used for both task performance and communication.

Understanding appropriate robot design for physical/haptic human-robot interaction, from the mechanical to the algorithmic, requires amalgamated knowledge from a variety of fields outside traditional engineering/computer science, such as biomechanics, psychology, and neuroscience. Here, I will briefly examine several research topics that should be addressed in order to improve the haptic aspects of such human-robot physical collaborations.

Haptics is usually overloaded, but it can be cleaved. In scenarios such as human-robot collaborative furniture moving, the haptic interaction is overloaded. That is, haptic interactions are necessary to perform the physical task and can also be a means to communicate, sometimes unintentionally. If I move my end of the couch down because I need to readjust my grip, a robot or a human on the other side may interpret instead that I want to set the couch down. In conventional physical interactions, we have no choice but to conflate the operative and communicative/social aspects of haptics. But artificial haptic feedback provided to humans or haptic interaction signals interpreted by robots can cleave these dual purposes-we can intentionally break the closed-loop nature of real-world physical interactions. For humans, this could mean using natural physical interactions for task performance and also wearing body-mounted tactile displays/sensors to receive and send communications (Figure 1). For robots, this means combining rich, multimodal observations of the environment and behavior of the human to determine appropriate actions. It is important to recognize that even though humans do not pair to perform such tasks seamlessly, we expect robots to do better in interacting with humans.

Haptics can solve communication bottlenecks, but we need to circumvent inherent limitations. Haptics may be able to communicate certain types of information more quickly than sight or sound; for example, a force vector immediately conveys both direction and magnitude. In addition, in environments where other displays would be disruptive, the targeted and private nature of haptic feedback can be an advantage. Yet, the classic manner in which a force vector is conveyed by haptics is using a kinesthetic, desktop-mounted device with relatively large motors, cumbersome linkages, and small workspace. This presents many challenges in haptic device design that must take into account wearability and the capabilities of human haptic sensing. Foremost is that the amount of information that can be transmitted through touch is limited in large part by the location and distribution of human mechanoreceptors (sensors embedded in the skin). Not surprisingly, many haptic devices are designed to be held or worn at the fingertips, which have very 

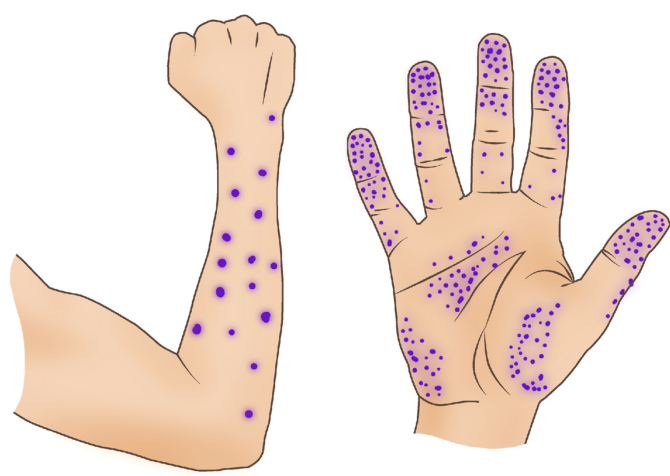

Fig. 2. The density and types of mechanoreceptors (touch sensors embedded in the skin) differ greatly between the glabrous skin on the hand and the hairy skin on the arm. To leave the hand free for activities of daily living, wearable haptic devices will need to be able to communicate information through other parts of the body, taking into account bottlenecks in human haptic sensing and perception. Figure courtesy of Evan Pezent.

high density of mechanoreceptors (Figure 2). Such haptic devices can render the hand useless for other tasks. We need to understand the amount and type of information that can be transmitted via haptics to other parts of the body, ranging from the wrist to the bottom of the foot.

Understanding and creating affective haptics will be essential for social robots. Touch is the primary nonverbal means of communication of emotion between humans. Humans use a variety of different gestures to convey emotions, including squeezes, pats, and strokes; for example, a handshake is used to say hello and a gentle pat is used to express encouragement. Both our physical and emotional wellbeing rely on human-human touch; it is reasonable to expect that human-robot touch will be important for usability and acceptance of many kinds of robots. Many haptic devices have been designed for social touch and seek to replicate specific interactions or gestures, such as a hug. Human-robot interactions generally need to be more flexible, requiring a better understanding of both how social touches should be performed and what hardware and software implementations are needed to achieve them.

There is great potential for haptic devices to allow touch-based interaction between humans and robots - enabling communication in a salient but private manner that frees other sensory channels. We can use the direct physical interaction between humans and robots to achieve haptic communication, although new haptic devices have the opportunity to separate the physical interaction from communications. For haptic devices to become ubiquitous for such purposes, they must be intuitive, unobtrusive, wearable, and socially acceptable.

Received March 2018; accepted April 2018 